\title{
Correction: Do Cardiac Rehabilitation Affect Clinical Prognoses Such as Recurrence, Readmission, Revascularization, and Mortality After AMI?: Systematic Review and Meta-Analysis
}

\author{
Chul Kim, MD, $\mathrm{PhD}^{1}$, Insun Choi, $\mathrm{PhD}^{2}$, Songhee Cho, $\mathrm{PhD}^{2}$, Ae Ryoung Kim, $\mathrm{MD}^{3}$,
} Wonseok Kim, $\mathrm{MD}, \mathrm{PhD}^{4}$, Sungju Jee, $\mathrm{MD}, \mathrm{PhD}^{5,6,7}$

${ }^{1}$ Department of Rehabilitation Medicine, Inje University Sanggye Paik Hospital, Seoul;

${ }^{2}$ National Evidence-based Healthcare Collaborating Agency, Seoul;

${ }^{3}$ Department of Rehabilitation Medicine, Kyungpook National University Hospital, Daegu;

${ }^{4}$ Department of Rehabilitation Medicine, Seoul National University Bundang Hospital, Seongnam;

${ }^{5}$ Department of Rehabilitation Medicine, Chungnam National University College of Medicine, Daejeon;

${ }^{6}$ Daejeonchungcheong Regional Medical Rehabilitation Center, Chungnam National University Hospital, Daejeon;

${ }^{7}$ Dajeonchungcheong Regional Cardiocerebrovascular Center, Chungnam National University Hospital, Daejeon, Korea

https://doi.org/10.5535/arm.20080

Ann Rehabil Med 2021;45(1):57-70

After publication of the article, we found the fifth author name was published incorrectly. "Wonseok Kim" was changed to "Won-Seok Kim." The correct author list is:

\section{Chul Kim, MD, $\mathrm{PhD}^{1}$, Insun Choi, $\mathrm{PhD}^{2}$, Songhee Cho, $\mathrm{PhD}^{2}$, Ae Ryoung Kim, $\mathrm{MD}^{3}$, Won-Seok Kim, $\mathrm{MD}, \mathrm{PhD}^{4}$, Sungju Jee, $\mathrm{MD}, \mathrm{PhD}^{5,6,7}$}

(c) This is an open-access article distributed under the terms of the Creative Commons Attribution Non-Commercial License (http://creativecommons.org/ licenses/by-nc/4.0) which permits unrestricted noncommercial use, distribution, and reproduction in any medium, provided the original work is properly cited. Copyright ( 2021 by Korean Academy of Rehabilitation Medicine 